\title{
DYNAMIC LOAD BALANCING IN CLOUD COMPUTING PLATFORM
}

\author{
N. A. Joshi \\ Professor, Department of MCA, Dharmsinh Desai University
}

\begin{abstract}
The cloud computing platform has played significant role behind the exponential growth of modern IT industry. More and more organizations \& individuals from various sectors of society are directory or indirectly consuming cloud computing platform. Such rising demand for cloud based computing resources has generated requirement of optimized resource management and load balancing. Extensive research work is taking place for efficient resource management and load balancing. A dynamic approach for virtual machine load balancing is presented here. The load balancing mechanism presented here offers better efficiency due to its multi-threaded approach.
\end{abstract}

Key words: load balancing, cloud computing, collaborated cloud, task migration.

Cite this Article: N. A. Joshi, Dynamic Load Balancing in Cloud Computing Platform, International Journal of Computer Engineering and Technology 10(3), 2019, pp. 39-44.

http://iaeme.com/Home/issue/IJCET?Volume=10\&Issue=3

\section{INTRODUCTION}

Innovative headways in storage technologies, internet of things and communication technologies has given momentum to usage of cloud computing platform. Cost effectiveness, negligible maintenance and uninterrupted availability are some of the driving forces behind the fast growth and usage of cloud computing technologies. Moreover various open source cloud computing platforms also have started getting handsome attraction in the cloud computing market. Various emerging technologies such as edge computing and data analytic techniques such as machine learning and deep learning also are key players in growth of cloud computing.

On other side, the exponential demand for cloud based resources often creates problems on resource management. Utilization of cloud based resources in the most cost effective way is of course one of the primary requirement for service consumers. Moreover, cloud service providers also often stretch on optimum resource management by means of load balancing [9] for sake of meeting the Service Level Agreements.

Recent advancements in cloud load balancing are more towards collaborated clouds and load balancing in such cloud computing environments. An improved work on load balancing 
technique [7] is suggested in this paper. Survey on related load balancing work is described in section 2. Section 3 presents the suggested mechanism. Outcomes are presented in section 4.

\section{RELEATED WORK}

Chien et al. have proposed a load balancing technique which is based on job completion time. The technique helps in increasing performance in line with the job processing time [3]. On other side, Papadopoulos has proposed a resource management technique for balancing workload of cloud servers. The technique works on control-based self-adaptive randomized optimization [11]. Vasudevan et al. have suggested a honey bee theory based technique that assigns available resources to cloud network. The technique works on or reducing service makespan [12].

Nan S. et. al. have suggested a technique based on enhancement of annealing algorithms for efficient placement of virtual machines. The technique works on resource utilization, dynamic placement and optimized annealing [10]. Zhu et al. have suggested a load balancing technique which is based on improved Particle Swarm Optimization [13]. N. Joshi et. al. have suggested prototype mechanism for load balancing in cloud computing environment. The technique works on centralized load balancing approach. The suggested mechanism works on relocating tasks instead of migrating whole virtual machine [6].

Ajit et. al. have suggested a resource management strategy for reducing response time. The mechanism works on weighted signature and collects value for load assignment for available nodes. Based on the weighted signatures, the mechanism maps virtual machines to physical machines [1]. A load sharing mechanism proposed by Azar et. al. works on first fit based strategy and f-restricted algorithm [2]. Kapur has presented a load balancing technique for reserved and on-demand cloud resources [8].

N. Joshi has proposed a resource management technique for dynamic load balancing for collaborated cloud computing environments [5]. Deng et. al. have suggested a technique that applies optimized power management and resource management by means of an EcoPower algorithm. The technique works on basis of Lyapunov optimization [4].

Different load balancing approaches are applied for optimized resource utilization. Some load balancing techniques are based on diverting the incoming requests to other under loaded virtual machines. Whereas, some other techniques exist which deal with migration of entire overloaded virtual machine to other physical machine. Some techniques offer intra-cloud load balancing whereas few others offer inter-cloud load balancing. An optimized version of a technique suggested in [7] is presented here.

\section{MECHANISM}

The suggested improved mechanism functions in three threads: thread_OverLoadedVM, thread_UnderLoadedVM and thread_LoadBalancer.

The threads thread_UnderLoadedVM and thread_OverLoadedVM are responsible to fill in the queues qUnderloaded and qOverloaded to hold a set of under loaded and overloaded virtual machines respectively. Block diagram is shown in figure 1.

The time intervals $\mathrm{Tu}$ and To represent snooze time duration for the threads thread_UnderLoadedVM and thread_OverLoadedVM respectively. Moreover, appropriate mutual exclusion tools also have been incorporated in order to prevent starvation and inconsistency.

Module LoadBalancer

\{ 
int $\mathrm{Tu}, \mathrm{To}$;

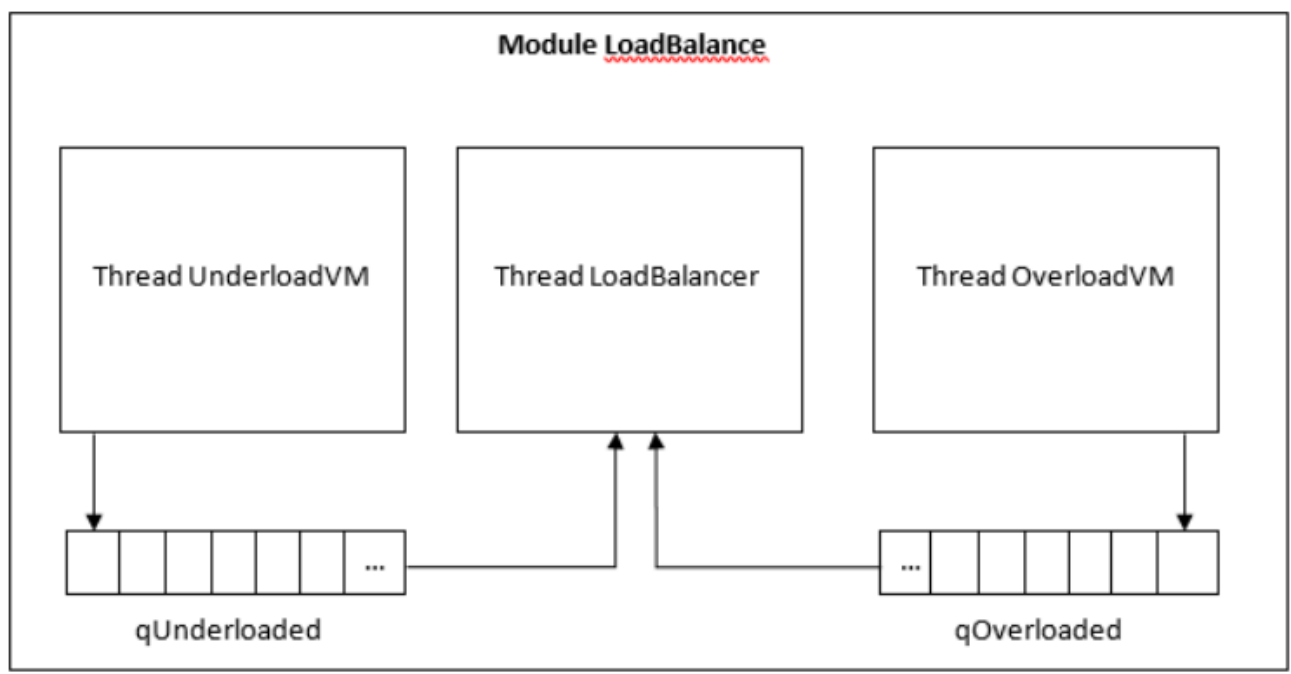

Figure 1: Load balancer module

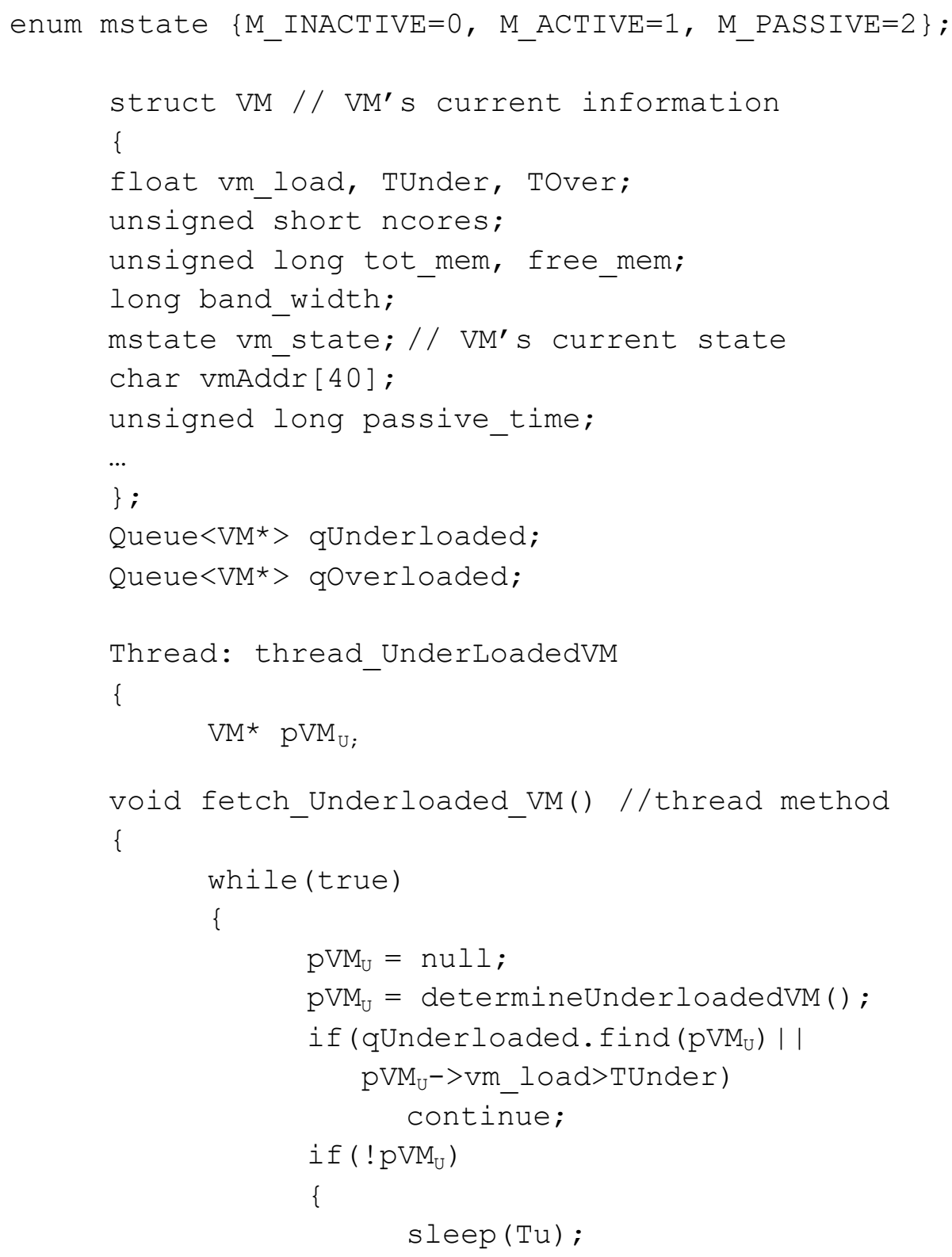


N. A. Joshi

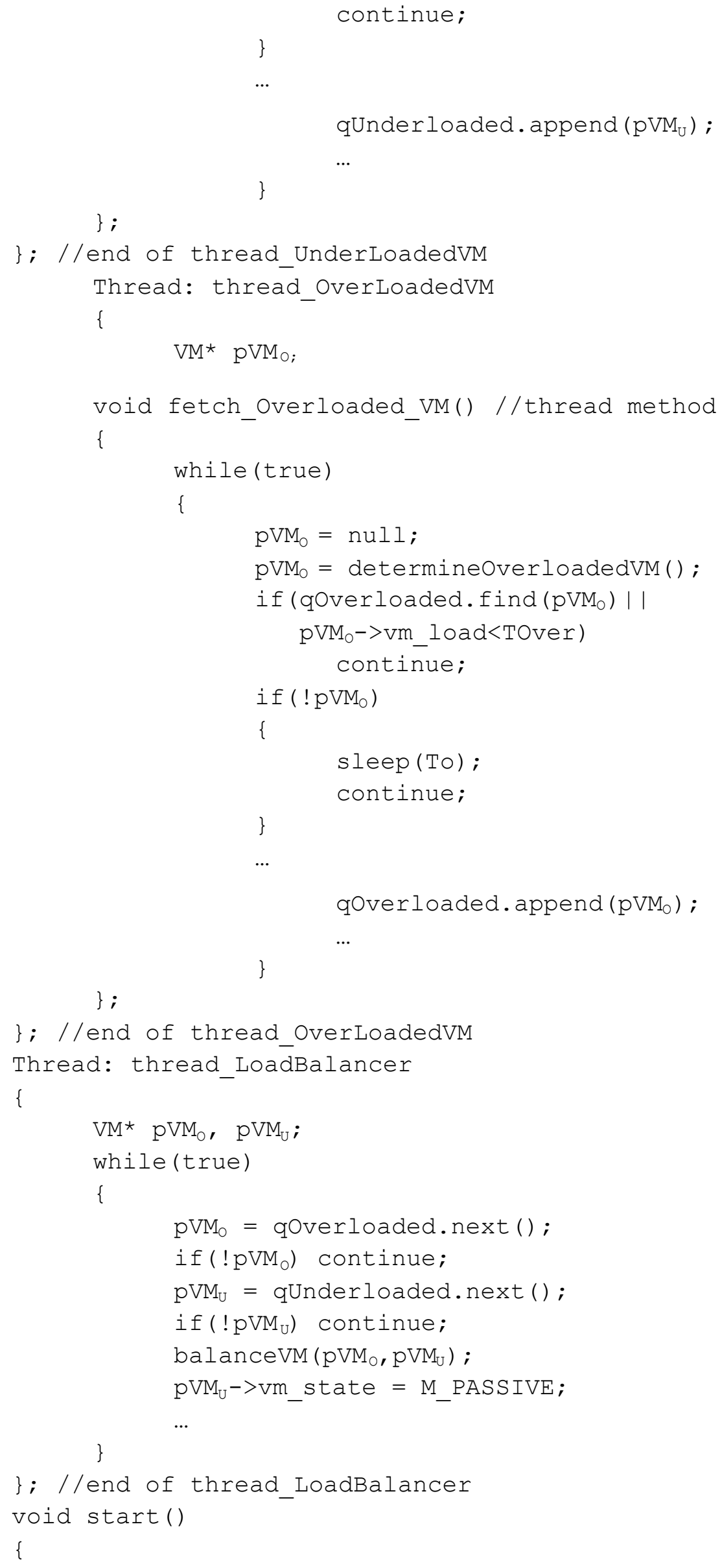




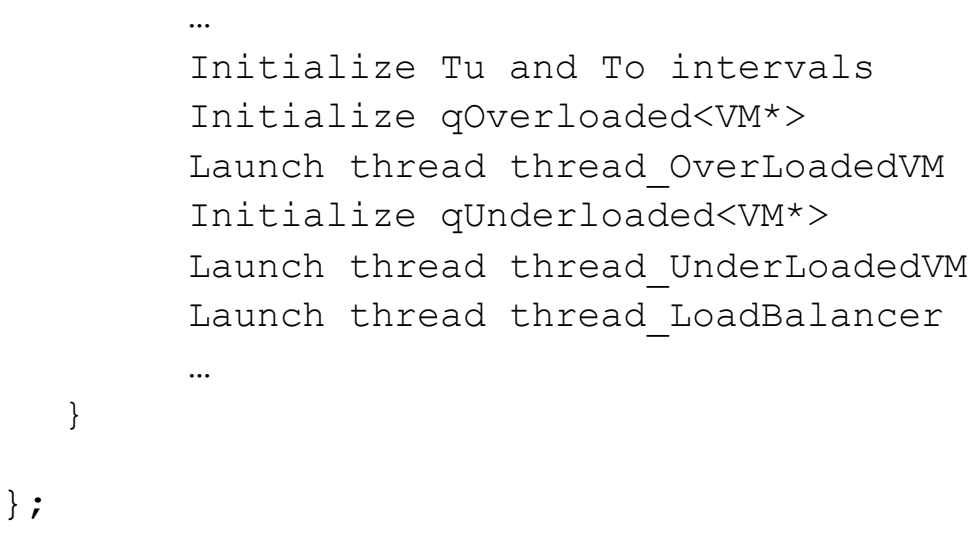

\section{OUTCOMES}

The thread thread_LoadBalancer is responsible to carry out load balancing work among the virtual machines listed in qOverloaded and qunderloaded. The thread thread_LoadBalancer achieves its objective means of shifting workload from the overloaded virtual machine to under loaded virtual machine.

The threaded approach helps the program threads to function independently which causes faster execution of overall mechanism. Moreover, the suggested mechanism has been designed with reference to necessary aspects for mutual exclusion and synchronization.

\section{CONCLUSION}

Efficient resource management and load balancing plays vital role in cloud computing environments. This paper describes a load balancing technique. The suggested load balancing mechanism works on collaboration of three simultaneously working threads, which helps in faster execution of the mechanism itself. Moreover, the load balancer is no more required to wait for availability of information about concerned heavily loaded and lightly loaded virtual machines. The work may be extended in future on flexibility of passive time duration of individual virtual machine. Moreover, the workload status may be determined at time of actual load balancing.

\section{REFERENCES}

[1] Ajit, M., Vidya, G., VM level load balancing in cloud environment, IEEE Fourth International Conference on Computing, Communications and Networking Technologies, July 2013

[2] Azar, Y., Cohen, I.R., Fiat, A., Roytman, A., Packing small vectors, Proceedings of the Twenty-Seventh Annual ACM-SIAM Symposium on Discrete Algorithms, January 2016

[3] Chien, N. K., Son, N. H., Loc, H. D., Load balancing algorithm based on estimating finish time of services in cloud computing, 18th IEEE International Conference on Advanced Communication Technology, January, 2016

[4] Deng, Y., Lau, R.W., Dynamic load balancing in distributed virtual environments using heat diffusion, ACM Transactions on Multimedia Computing, Communications, and Applications, 10 (2), 2014

[5] Joshi, N., Load Balancing in Cloud Computing Using Process Migration, International Journal of Advanced Research in Engineering and Technology, 5(4), April, 2014 
[6] Joshi, N., Choksi, D. B., Kotecha, K. and Pandya S., Implementation of Novel Load Balancing Technique in Cloud Computing Environment, International Conference on Computer Communication and Informatics, January, 2018

[7] Joshi, N., Improved Load Balancing in Cloud Computing Environments, International Journal of Research in Electronics and Computer Engineering, 6(4), December, 2018

[8] Kapur, R., A workload balanced approach for resource scheduling in cloud computing, Eighth IEEE International Conference on Contemporary Computing, August, 2015

[9] Moges, F. F. and Abebe, S. L., Energy Aware VM Placement Algorithms for the OpenStack Neat Consolidation Framework, Journal of Cloud Computing Advances, Systems and Applications, January, 2019

[10] Nan, S., Shi, A., Chen, C., Chen, E. and Wang, Y., Research on Virtual Machine Placement in the Cloud Based on Improved Simulated Annealing Algorithm, World Automation Congress, 2016

[11] Papadopoulos, A. V., Klein, C., Maggio, M., Drango, J., Dellkrantz, M., Hernndez, F. and Arzn, K. E., Control-based load-balancing techniques: Analysis and performance evaluation via a randomized optimization approach. Control Engineering Practice, 52, 2016

[12] Vasudevan, S. K., Anandaram, S., Menon, A. J. and Aravinth, A., A novel improved honey bee based load balancing technique in cloud computing environment, Asian Journal of Information Technology, 15 (9), 2016

[13] Zhu, Y., Zhao, D., Wang, W. and He, H., A Novel Load Balancing Algorithm Based on Improved Particle Swarm Optimization in Cloud Computing Environment. International Conference on Human Centered Computing, Springer, January, 2016 